\title{
FITOSSOCIOLOGIA DO SUB-BOSQUE DO PARQUE AMBIENTAL RUBENS DALLEGRAVE, IRATI, PR
}

\author{
Andréia Pimentel*, Vagner Putton**, Luciano Farinha Watzlawick***, \\ Álvaro Felipe Valério***, Daniel Saueressig**** \\ *Enga . Florestal, Mestranda em Manejo Florestal, UFPR - andreia_pimentell@hotmail.com \\ **Eng. Florestal, REMASA - vagner@remasa.com.br \\ ***Eng. Florestal, Dr., UNICENTRO - farinha@unicentro.br - alvarofvalerio@yahoo.com.br \\ ****Acadêmico de Engenharia Florestal, UNICENTRO - floraconsultoria@gmail.com \\ Recebido para publicação: 06/02/2007 - Aceito para publicação: 11/12/2007
}

\begin{abstract}
Resumo
Este estudo foi realizado em um trecho de Floresta Ombrófila Mista secundária do Parque Ambiental Rubens Dallegrave e analisou a florística e a estrutura dos indivíduos arbóreos e arbustivos da regeneração da comunidade. Foram utilizadas 14 unidades amostrais para cada componente, identificando-se e contando-se os indivíduos com DAP $\leq 10 \mathrm{~cm}$, divididos em três unidades: a regeneração com altura inferior a $0,5 \mathrm{~m}$, a regeneração intermediária, entre $0,5 \mathrm{~m}$ e $2 \mathrm{~m}$ de altura, em parcelas de $1 \mathrm{~m}^{2}$ e $9 \mathrm{~m}^{2}$, respectivamente, e a identificação e mensuração dos indivíduos com altura acima de $2 \mathrm{~m}$ em parcelas com $100 \mathrm{~m}^{2}$. Para a regeneração inferior a $0,5 \mathrm{~m}$ de altura, verificou-se a densidade de 27.857 ind.ha $^{-1}$, pertencentes a 6 famílias e 10 espécies, tendo maior freqüência Cupania vernalis, Matayba elaeagnoides e Nectandra megapotamica. Para a regeneração que compõe o subbosque intermediário, verificou-se a densidade de 16.066 ind.ha $^{-1}$ de 20 famílias, 28 espécies, tendo maior freqüência Cupania vernalis, Nectandra megapotamica e Matayba elaeagnoides. O componente do sub-bosque superior a $2 \mathrm{~m}$ apresentou densidade de 864 ind.ha $^{-1}$, pertencentes a 13 famílias e 21 espécies, sendo as de maior freqüência e VI Cupania vernalis, Lonchocarpus muehlbergianus e Nectandra megapotamica.

Palavras-chave: Regeneração natural; análise florística; estrutura da regeneração.
\end{abstract}

\begin{abstract}
Understory structure of Rubens Dallegrave environmental park, Irati, Paraná. This research was developed in a secondary Subtropical Ombrophilous Forest in Rubens Dallegrave Environmental Park, and analyzes the floristic of the arboreal and structure of regeneration. Fourteen plots were located at each component, comprehending the identification and count of individuals with DAP $\leq 10$ $\mathrm{cm}$, divided in three unities, being counted and identified the regeneration smaller than $0,5 \mathrm{~m}$ height and the intermediate between $0,5 \mathrm{~m}$ and $2 \mathrm{~m}$ height, in plots of $1 \mathrm{~m}^{2}$ and $9 \mathrm{~m}^{2}$ respectively, and identification and measurement of individuals more than $2 \mathrm{~m}$ height in subplots with $100 \mathrm{~m}^{2}$. For the regeneration smaller than $0,5 \mathrm{~m}$ it was verified a density of 7.857 ind.ha $^{-1}$, composed by 6 families of 10 species, being the most frequent: Cupania vernalis, Matayba elaeagnoides and Nectandra megapotamica. For the understory regeneration, it was verified a density was 16.066 ind.ha $^{-1}$ with 20 families, and 28 species, being the most frequent Cupania vernalis, Nectandra megapotamica and Matayba elaeagnoides. The sub-wood over than $2 \mathrm{~m}$, showed density of 864 ind.ha $^{-1}$, represented by 13 families and 21 species, being the most frequent and Importance Value: Cupania vernalis, Lonchocarpus muehlbergianus and Nectandra megapotamica.

Keywords: Natural regeneration; floristic analyze; structure of regeneration.
\end{abstract}

\section{INTRODUÇ̃̃O}

A regeneração natural é a forma mais antiga de renovação de uma floresta. Todas as espécies arbóreas possuem mecanismos que permitem sua perpetuação no ambiente. A regeneração natural é elemento importante na evolução de uma espécie arbórea e está intimamente relacionada com o ambiente em que a espécie se desenvolveu, assim como com a biocenose em que evoluiu (SEITZ; JANKOVSKI, 1998). 
Para Amo Rodriguez; Gomez-Pompa (1976), a regeneração natural é o estágio que segue à independência da plântula da reserva da semente e precede o estado vegetativo adulto e o reprodutivo, ou seja, é o indivíduo jovem na floresta. Esse estágio é de grande importância, já que o fracasso dos processos adaptativos, nesse período, pode eliminar a espécie do ambiente.

De acordo com Gama et al. (2002), a regeneração natural refere-se às fases iniciais de estabelecimento e desenvolvimento das plantas. Sua boa condição quantitativa e qualitativa possibilita a preservação, a conservação e a formação de florestas, tanto de proteção integral como de uso sustentável. $\mathrm{O}$ entendimento dos processos de regeneração natural de florestas passa pelo conhecimento de informações básicas de caracterização da vegetação.

Segundo Lamprecht (1990), o tipo de regeneração surge na dependência de numerosas précondições, que são bastante diversas de uma espécie arbórea para outra. Em todos os casos, são indispensáveis as seguintes condições: presença em quantidade suficiente de sementes viáveis e condições edafo-climáticas à altura das exigências de germinação e crescimento.

Os elementos mais importantes na avaliação da regeneração natural são a densidade e a distribuição das plantas. Isso porque a nova população deve ter um número suficiente de plantas por unidade de área, além de apresentar uniformidade na distribuição, de forma que não se apresentem grandes áreas vazias. Em estágios mais desenvolvidos, geralmente a partir do segundo ano, avaliam-se também o desenvolvimento e a qualidade dos indivíduos (STEIN, 1974).

Carvalho (1982) esclarece que a análise da estrutura da regeneração fornece a relação e a quantidade de espécies que constituem o estoque da floresta, suas dimensões e sua distribuição na comunidade vegetal. Hosokawa; Solter (1995) complementam afirmando que os resultados das análises estruturais permitem fazer deduções sobre as origens, características ecológicas e sinecológicas, dinamismo e tendências de seu desenvolvimento.

Verifica-se a necessidade da realização de estudos que retratem a realidade da floresta, no que tange à florística e sua estrutura, para que essas informações possam ser úteis na elaboração e planejamento de ações que objetivem a conservação ou mesmo a recuperação da formação florestal, procurando retratar a sua diversidade ao máximo (WATZLAWICK et al., 2005).

Vários estudos sobre a composição florística e as relações fitossociológicas do componente arbustivo-arbóreo em florestas naturais apontam a regeneração natural como importante estratégia de reabilitação e recuperação de áreas perturbadas (NAPPO et al., 2004).

O presente estudo teve como objetivo conhecer a diversidade florística e analisar a estrutura da regeneração de um trecho de Floresta Ombrófila Mista no Parque Ambiental Rubens Dallegrave, no município de Irati, estado do Paraná.

\section{MATERIAIS E MÉTODOS}

\section{Caracterização da área}

O município de Irati localiza-se na região centro-sul do estado do Paraná (2527'56" S; $50^{\circ} 37^{\prime} 51^{\prime}$ W), a $156 \mathrm{~km}$ de Curitiba, na sub-região dos pinhais do Segundo Planalto (Figura 1).

Em relação ao tempo geológico, o solo pertence ao permiano carbonífero, com topografia de forte ondulada a acidentada, solos acinzentados/vermelhos ao norte e castanhos ao sul. A geologia e a paleontologia asseguram que a região, há mais de 250 milhões de anos, foi fundo de mar. Os tipos de solos predominantes são o Podzólico Vermelho Amarelo (MAACK, 1968), ou, segundo a classificação da Embrapa (1999), Argissolo Amarelo distrófico e Cambissolo.

$\mathrm{O}$ clima regional è do tipo $\mathrm{Cfb}$, de acordo com a classificação de Köppen, apresentando estações climáticas bem definidas, com chuvas distribuídas durante todo o ano, com geadas severas e com umidade relativa do ar acentuada. A precipitação média anual fica entre 1500 e 1600 mm (SUDERHSA, 1998). A temperatura média anual é de aproximadamente $18{ }^{\circ} \mathrm{C}$, com mínima de $-2{ }^{\circ} \mathrm{C}$ e máxima de 32 ${ }^{\circ} \mathrm{C}$. O limiar médio das temperaturas de Irati varia entre 13 e $23,5^{\circ} \mathrm{C}$ (SIMEPAR, 2006).

A área, de 9,7 ha, está sob domínio da Floresta Ombrófila Mista, sendo esta representada por vegetação secundária em estágio médio e inicial de regeneração. da cidade.

No centro da área há um curso d'água que desemboca numa cachoeira, um dos pontos turísticos 


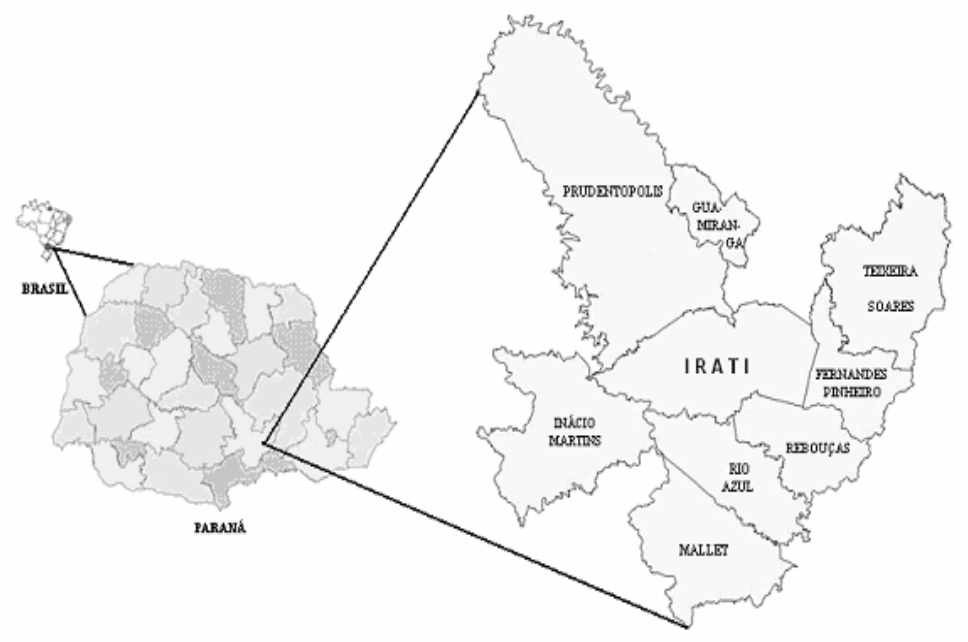

Figura 1. Localização regional do município de Irati.

Figure 1. Regional location of the Irati county.

\section{Metodologia}

As unidades amostrais foram locadas sistematicamente em coordenadas UTM e o tamanho da parcela definido de acordo com o componente da regeneração a ser analisado, contemplando toda a variabilidade morfológica e estrutural possível da vegetação na área. A distribuição das unidades amostrais está representada na figura 2.

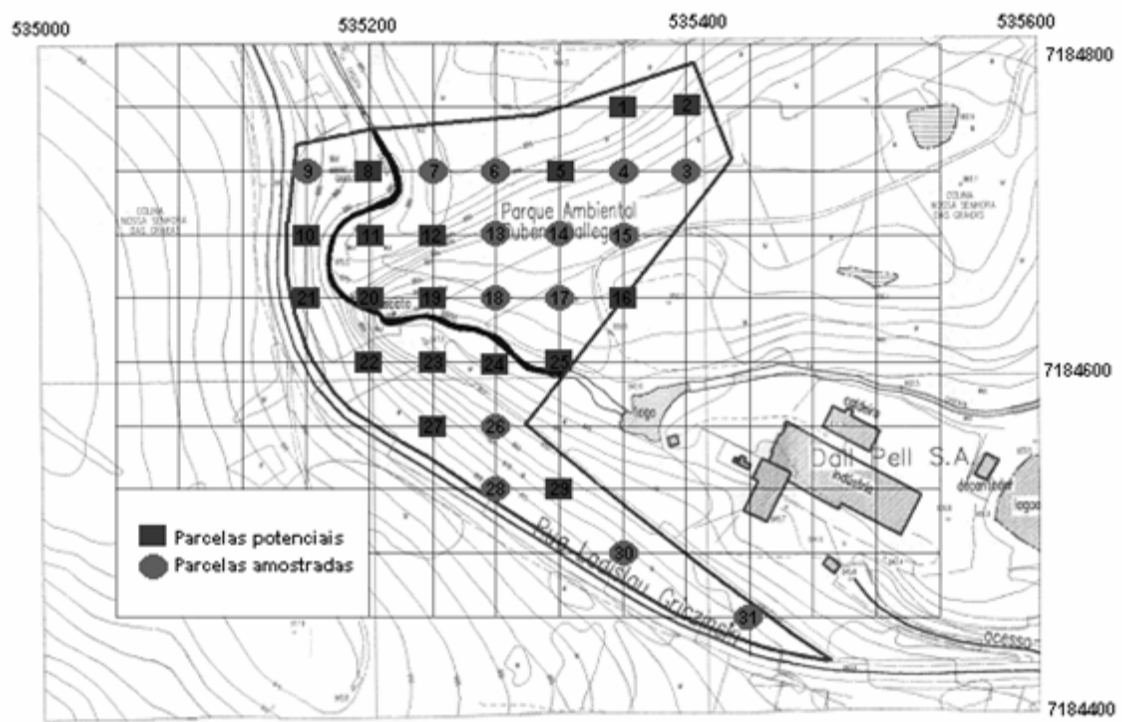

Escala 1:10.000

Figura 2. Área de estudo e disposição sistemática das unidades potenciais e as unidades amostradas na área de estudo.

Figure 2. Study area and systematic arrangement of the potential units and the sampled units.

Para uma inferência acurada do sub-bosque, ele foi dividido em três estratos - superior, intermediário e inferior -, com unidades amostrais de $100 \mathrm{~m}^{2}, 9 \mathrm{~m}^{2}$ e $1 \mathrm{~m}^{2}$, respectivamente, destinadas à avaliação da composição do sub-bosque com diâmetros menores que $10 \mathrm{~cm}$, divididas em três classes de 
alturas: para o estrato inferior, menor que $0,5 \mathrm{~m}$; para o intermediário, entre 0,5 e $2 \mathrm{~m}$; para o estrato superior, altura maior que $2 \mathrm{~m}$ (conforme apresentado na Figura 3).

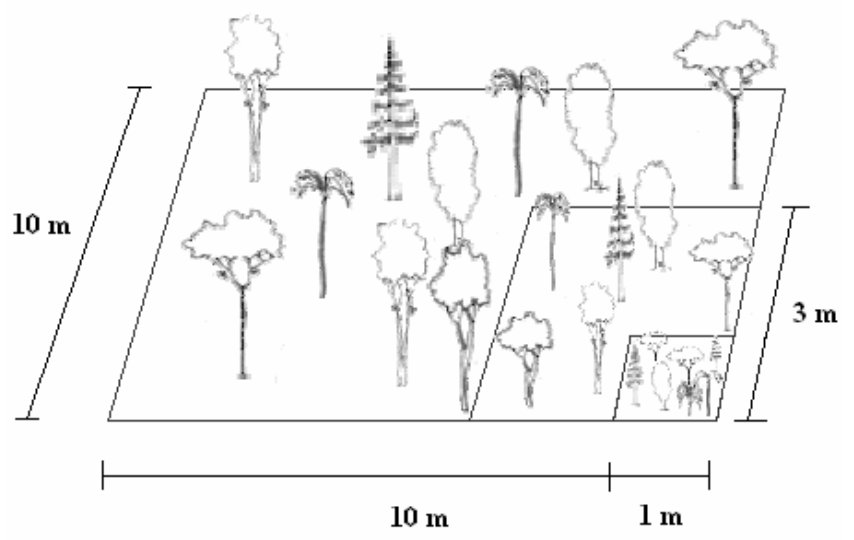

Figura 3. Representação esquemática das unidades amostrais realizadas.

Figure 3. Schematic representation of sample units.

A identificação das plantas em nível de famílias e espécies segue o sistema de classificação de Cronquist (1988).

\section{RESULTADOS E DISCUSSÕES}

A curva espécie/área representa o acréscimo de espécies do sub-bosque superior (altura $\geq 2 \mathrm{~m}$ ) em relação à área de amostragem (Figura 4). Nota-se que, a partir de $900 \mathrm{~m}^{2}$ de área amostral, ou seja, após a unidade amostral nove, ocorre a estabilização da curva. Isso indica que as 14 unidades utilizadas foram suficientes para caracterizar a vegetação em estudo, em termos de composição de espécies.

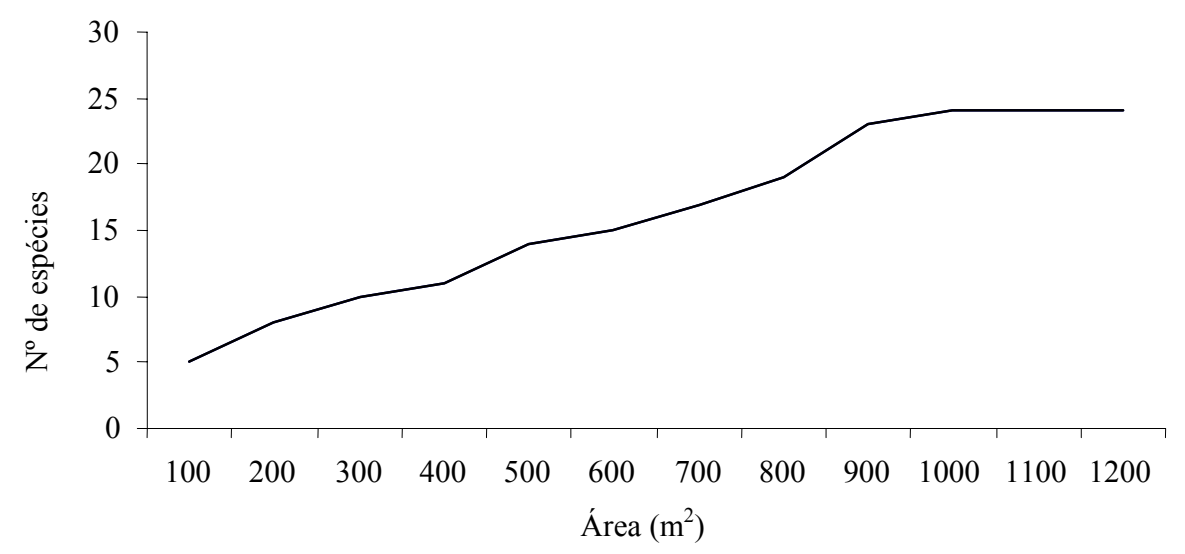

Figura 4. Curva do número de espécies/área, para verificação da suficiência amostral do sub-bosque superior em um fragmento de Floresta Ombrófila Mista.

Figure 4. Curve species/area for the sample sufficiency of the understory in a fragment of Subtropical Ombrophilous Forest.

No sub-bosque superior, foram mensurados 121 indivíduos, distribuídos em 13 famílias e 23 espécies (Tabela 1). A densidade e dominância total estimada por hectare foi de 864 indivíduos e de 3,74 $\mathrm{m}^{2}$, respectivamente. 
Tabela 1. Espécies amostradas no inventário florestal do sub-bosque superior (altura $>2 \mathrm{~m}$ ) de um trecho de Floresta Ombrófila Mista Secundária.

Table 1. Sampled species in the forest inventory of the high understory (height $>2 \mathrm{~m}$ ) in a fragment of Subtropical Ombrophilous Forest.

\begin{tabular}{llc}
\hline Família & Nome Científico & Nome Popular \\
\hline Anonaceae & Rollinia rugulosa Schlecht. & Ariticum-de-porco \\
Araliaceae & Oreopanax fulvum Marchal & Embauvarana \\
Clethraceae & Styrax leprosus Hook. \& Arn. & Carne-de-vaca \\
Euphorbiaceae & Actinostemon concolor (Spreng.) Müll. Arg. & Laranjeira-do-mato \\
Euphorbiaceae & Sebastiania brasiliensis Spreng. & Leiterinho \\
Euphorbiaceae & Sapium glandulatum (Vell.) Pax & Leitero \\
Fabaceae & Myrocarpus cf. frondosus Allemão & Cateretê \\
Fabaceae & Machaerium paraguariense Hassl. & Cateretê \\
Fabaceae & Machaerium stipitatum (D.C.) Vogel & Sapuva \\
Fabaceae & Lonchocarpus muehlbergianus Hassl. & Timbó \\
Flacourtiaceae & Casearia sylvestris Sw. & Cafezeiro \\
Lauraceae & Nectandra megapotamica (Spreng.) Mez & Canela Imbuia \\
Meliaceae & Cedrela fissilis Vell. & Cedro \\
Mimosaceae & Albizia cf. polycephala (Benth.) Killip ex Record & Farinha-seca \\
Mimosaceae & Inga sp. & Ingá \\
Mimosaceae & Eupatorium sp. & Vassourão \\
Rhamnaceae & Hovenia dulcis Thunb. & Uva-do-japão \\
Sapindaceae & Cupania vernalis Cambess. & Cuvatã \\
Sapindaceae & Matayba elaeagnoides Radlk. & Miguel-pintado \\
Sapindaceae & Allophylus edulis (A. St.-Hil., Cambess. \& A. Juss.) Radlk. & Vacum \\
Solanaceae & Solanum sanctae-katharinae Dunal in D.C. & Canema \\
Solanaceae & Cestrum sp. & Cestrum \\
Tiliaceae & Luehea divaricata Mart. & Açoita-cavalo \\
\hline
\end{tabular}

A família de maior importância e riqueza florística foi Fabaceae (4), seguida de Euphorbiaceae, Mimosaceae e Sapindaceae (3). Considerando estudos do sub-bosque dos bracatingais realizados por Mazza et al. (2000) em tipologia florestal pertencente à área de domínio da Floresta de Araucária com influência da Floresta Atlântica, as famílias que tiveram maior destaque foram Fabaceae, Agavaceae, Sapindaceae e Lauraceae, muito semelhante aos resultados da formação vegetal em estudo. Para Barddal et al. (2004), as famílias mais relevantes foram Myrtaceae, Sapindaceae, Euphorbiaceae e Rubiaceae. Porém, em estudos sobre riqueza de sub-bosque de Floresta Ombrófila Mista realizado por Liebsch e Acra (2004) em Tijucas do Sul, Paraná, não se verificou grande importância florística para essas famílias.

Destacaram-se para o estrato superior, pela maior densidade, Cupania vernalis (186 ind.ha ${ }^{-1}$ ), Lonchocarpus muehlbergianus (121 ind.ha $\left.{ }^{-1}\right)$ e Nectandra megapotamica (107 ind.ha $\left.{ }^{-1}\right)$. As seis espécies de maior valor de importância desse estrato foram: Cupania vernalis (18,93\%), Lonchocarpus muehlbergianus (13,33\%), Nectandra megapotamica (10,30\%), Allophylus edulis (8,96\%), Matayba elaeagnoides (4,63\%) e Styrax leprosus $(4,49 \%)$, totalizando $61 \%$ do VI total da amostra. Os valores dos parâmetros fitossociológicos das espécies estão apresentados na tabela 2.

$\mathrm{O}$ índice de diversidade de Shannon e Weaver ( $\left.\mathrm{H}^{\prime}: 2,68\right)$ indica uma boa heterogeneidade florística do sub-bosque superior. Assim, tal diversidade de espécies revela uma relativa importância ecológica da floresta, que por sua vez está localizada em Área de Preservação Permanente (APP). O índice de diversidade de Shannon foi 2,49 para Barddal et al. (2004), em sub-bosque de uma Floresta Ombrófila Mista Aluvial, inferior ao resultado encontrado neste trabalho.

Para o estrato intermediário, as dez espécies com relevante importância, em razão de suas elevadas densidades, foram as seguintes: Cupania vernalis (3852 ind.ha $^{-1}$ ), Nectandra megapotamica (2541 ind.ha ${ }^{-1}$ ), Matayba elaeagnoides (2377 ind.ha $^{-1}$ ), Allophylus edulis (1721 ind.ha $\left.{ }^{-1}\right)$, Lonchocarpus muehlbergianus (656 ind.ha $\left.{ }^{-1}\right)$, Styrax leprosus (656 ind.ha $\left.{ }^{-1}\right)$, Cestrum sp. (492 ind.ha $\left.{ }^{-1}\right)$, Rollinia rugulosa $\left(492\right.$ ind.ha $\left.^{-1}\right)$, Campomanesia xanthocarpa $\left(410\right.$ ind.ha $\left.^{-1}\right)$, Citronella paniculata $\left(328\right.$ ind.ha $\left.^{-1}\right)$. 
Tabela 2. Relação das espécies arbóreas amostradas em sub-bosque superior com seus respectivos parâmetros fitossociológicos: N - Número de indivíduos, D - Densidade Absoluta (ind.ha ${ }^{-1}$ ), Do - Dominância Absoluta ( $\left.\mathrm{m}^{2} / \mathrm{ha}\right), \mathrm{FR}$ - Freqüência Relativa (\%), VC - Valor de cobertura (\%) e VI - Valor de importância (\%).

Table 2. Tree species sampled of high understory with structural parameters: $\mathrm{N}$ - number of trees, D Absolute density $\left(\right.$ ind $\left.\mathrm{ha}^{-1}\right)$, Do - Absolute dominance $\left(\mathrm{m}^{2} / \mathrm{ha}\right), \mathrm{FR}$ - relative frequency $(\%)$, $\mathrm{VC}$ - covering value (\%) e VI - importance value.

\begin{tabular}{lcccccc}
\hline Espécie & N & D & Do & FR & VC & VI \\
\hline Cupania vernalis & 26 & 186 & 0,86 & 12,28 & 22,26 & 18,93 \\
Lonchocarpus muehlbergianus & 17 & 121 & 0,58 & 10,53 & 14,73 & 13,33 \\
Nectandra megapotamica & 15 & 107 & 0,43 & 7,02 & 11,95 & 10,30 \\
Allophylus edulis & 11 & 79 & 0,34 & 8,77 & 9,06 & 8,96 \\
Matayba elaeagnoides & 6 & 43 & 0,20 & 3,51 & 5,19 & 4,63 \\
Styrax leprosus & 5 & 36 & 0,15 & 5,26 & 4,10 & 4,49 \\
Machaerium stipitatum & 4 & 29 & 0,14 & 5,26 & 3,50 & 4,09 \\
Machaerium paraguariense & 3 & 21 & 0,10 & 5,26 & 2,61 & 3,50 \\
Albizia cf. polycephala & 3 & 21 & 0,12 & 3,51 & 2,86 & 3,08 \\
Hovenia dulcis & 3 & 21 & 0,08 & 3,51 & 2,33 & 2,72 \\
Eupatorium sp. & 4 & 29 & 0,10 & 1,75 & 2,99 & 2,58 \\
Sebastiania brasiliensis & 3 & 21 & 0,12 & 1,75 & 2,80 & 2,45 \\
Morta & 2 & 14 & 0,06 & 3,51 & 1,66 & 2,28 \\
Solanum sanctae-catharinae & 2 & 14 & 0,06 & 3,51 & 1,60 & 2,24 \\
Sapium glandulatum & 2 & 14 & 0,05 & 3,51 & 1,51 & 2,18 \\
Inga sp. & 2 & 14 & 0,05 & 3,51 & 1,48 & 2,16 \\
Oreopanax fulvum & 2 & 14 & 0,04 & 3,51 & 1,34 & 2,06 \\
Luehea divaricata & 2 & 14 & 0,04 & 3,51 & 1,31 & 2,04 \\
Rollinia rugulosa & 3 & 21 & 0,06 & 1,75 & 2,10 & 1,98 \\
Cestrum sp. & 2 & 14 & 0,05 & 1,75 & 1,43 & 1,54 \\
Cedrela fissilis & 1 & 7 & 0,03 & 1,75 & 0,86 & 1,16 \\
Casearia sylvestris & 1 & 7 & 0,03 & 1,75 & 0,85 & 1,15 \\
Actinostemon concolor & 1 & 7 & 0,03 & 1,75 & 0,81 & 1,12 \\
Myrocarpus cf. frondosus & 1 & 7 & 0,02 & 1,75 & 0,67 & 1,03 \\
Total & 121 & 861 & 3,74 & 100 & 100 & 100 \\
\hline
\end{tabular}

Seguindo o mesmo conceito de classificação da importância das espécies do estrato intermediário, as espécies amostradas no estrato inferior foram: Cupania vernalis (7856 ind.ha ${ }^{-1}$ ), Matayba elaeagnoides (4286 ind.ha $\left.{ }^{-1}\right)$, Nectandra megapotamica (3570 ind.ha $\left.{ }^{-1}\right)$, Ocotea puberula (2856 ind.ha $\left.^{-1}\right)$, Styrax leprosus (2856 ind.ha $\left.{ }^{-1}\right)$, Eriobotrya japonica (2143 ind.ha $\left.{ }^{-1}\right)$, Machaerium stipitatum (1429 ind.ha $^{-1}$ ), Allophylus edulis (1429 ind.ha $\left.{ }^{-1}\right)$, Myrocarpus cf. frondosus (713 ind.ha $\left.{ }^{-1}\right)$, Trichilia elegans $\left(713\right.$ ind.ha $\left.^{-1}\right)$.

Por estar presente em todos os estratos do sub-bosque e alcançado o maior valor de importância, Cupania vernalis é considerada a principal espécie desse sub-bosque. Tal predominância dessa espécie em todos os estratos estudados se deve aos seguintes fatores: presença de boa quantidade de árvores matrizes e condições edafo-climáticas favoráveis. Dessa maneira, o processo de regeneração natural da espécie é beneficiado.

Os estudos desenvolvidos por Negrelle; Silva (1992) e Nascimento et al. (2001), em outros locais, em que se avaliou o componente com árvores de diâmetro maior ou igual a $10 \mathrm{~cm}$ na Floresta Ombrófila Mista, e por Longhi et al. (2000) em Floresta Estacional Decidual também demonstraram a grande importância da Cupania vernalis nessas tipologias florestais.

Para Barddal et al. (2004), Allophylus edulis (A. St.-Hil., Cambess. \& A. Juss.) Radlk. foi enquadrada como a principal espécie do sub-bosque, com o maior valor de importância.

A família mais abundante entre os regenerantes no sub-bosque foi Sapindaceae, na qual Cupania vernalis foi a mais presente (VC: 22,26\%), seguida por Fabaceae, na qual participam as espécies 
Lonchocarpus muehlbergianus, Myrocarpus cf. frondosus, Machaerium paraguariense, Machaerium stipitatum.

$\mathrm{Na}$ área inventariada foi constatada a presença de Hovenia dulcis Thunb., popularmente conhecida como uva-do-japão, espécie exótica com alto potencial invasivo. A ocorrência dessa espécie em Floresta Ombrófila Mista tem se tornado cada vez mais comum, pois os indivíduos apresentam frutificação consistente, regeneração natural intensa por sementes (CARVALHO, 2004), rápido crescimento e não dispõe de inimigo natural. Contudo, ela compromete a disseminação e prejudica o desenvolvimento de espécies autóctones, chegando até a substituí-las devido à sua agressividade e facilidade de adaptação.

Em se tratando de Floresta Ombrófila Mista, a composição florística poderia ser ainda mais diversa, com presença de araucária (Araucaria angustifolia) e espécies típicas de mata ciliar e florestas de encostas como, branquinho (Sebastiania commersoniana (Baill.) L.B. Sm. \& Downs), açoita-cavalo (Luehea divaricata Mart.) e monjoleiro (Parapiptadenia rigida (Benth.) Brenan), entre outras. Porém, há uma grande intervenção antrópica na área e grande presença de lianas, as quais dificultam o desenvolvimento natural da floresta.

\section{CONCLUSÕES}

São poucos os estudos realizados com o sub-bosque florestal, o que inviabiliza comparações com outras áreas dessa unidade fitogeográfica.

A alta densidade de indivíduos na estrutura de sub-bosque mensurada e identificada indica a auto-regeneração de tais espécies, apresentando condições propícias ao seu desenvolvimento e permanência na área.

Por ter alcançado o mais alto valor de importância e alta densidade, além de estar representada nos três estratos, Cupania vernalis Cambess. pode ser enquadrada como a espécie mais característica da regeneração natural da área estudada.

Com base no comportamento de Hovenia dulcis Thunb., pode-se supor o aumento populacional desta e de outras espécies invasoras, aumentando sua freqüência na floresta devido à contínua influência antrópica que o fragmento vem sofrendo.

\section{REFERÊNCIAS}

AMO RODRÍGUEZ, S. del; GÓMEZ-POMPA, A. Crecimento de estados juveniles de plantas em Selva Tropical Alta Perennifolia. In: GÓMEZ-POMPA, A.; VÁZQUEZ-YANEES, C.; AMO RODRÍGUEZ, S. del et al. Regeneration de Selvas. México: Continental, 1976. p. 549-565.

BARDDAL, M. L.; RODERJAN, C. V.; GALVÃO, F.; CURCIO, G. R. Fitossociologia do sub-bosque de uma Floresta Ombrófila Mista Aluvial, no município de Araucaria - PR. Ciência Florestal, Santa Maria, RS, v. 14, n. 1, p. 35-45, 2004.

CARVALHO, P. E. R. Espécies florestais brasileiras: recomendações silviculturais, potencialidades e uso da madeira. Brasília: EMBRAPA-CNPF; EMBRAPA-SPI, 1994. 640 p.

CARVALHO, P. E. R. Ecologia, silvicultura e usos da Uva-do-japão (Hovenia dulcis Thunberg). Colombo: EMBRAPA - CNPFlorestais, 1994. 11 p. (Circular Técnica, n. 23).

.CRONQUIST, A. The evolution and classification of flowering plants. $2^{\text {a }}$ ed. New York: The New York Botanical Garden, 1988. 555 p.

GAMA, J. R. V.; BOTELHO, S. A.; BETES-GAMA, M. M.; SCOLFORO, R. S. Estrutura e potencial futuro de utilização da regeneração natural de Floresta de Várzea Alta no município de Afuá, estado do Pará. Ciência Florestal, Santa Maria, RS, v. 13, n. 2, p. 71-82, 2002.

HOSOKAWA, R. T.; SOLTER, F. Manejo florestal. Curitiba: UFPR, 1995. 43 p.

LAMPRECHT, H. Silvicultura nos trópicos: ecossistemas florestais e respectivas espécies arbóreas possibilidades e métodos de aproveitamento sustentado. Eschborn: GTZ, 1990. 343 p. 
LIEBSCH, D.; ACRA, L. A. Riqueza de espécies de sub-bosque de um fragmento de Floresta Ombrófila Mista em Tijucas do Sul, PR. Ciência Florestal, Santa Maria, RS, v. 14, n. 1, p. 67-76, 2004.

LONGHI, S. J.; ARAUJO, M. M.; KELLING, M. B.; HOPPE, J. M.; MÜLlER, I.; BORSOI, G. A. Aspectos fitossociológicos de fragmento de Floresta Estacional Decidual, RS. Ciência Florestal, Santa Maria, RS,v. 10, n. 2, p. 59-74, 2000.

MAACK, R. 1968. Geografia física do estado do Paraná. Curitiba: BADEP/UFPR/IBPT. 350 p.

MAZZA, M. C. M. ; RODIGHIERI, H. ; NAKASHIMA, T. ; ZILLER, S. R. ; MAZZA, C.A.S. ; CONTO, A. J. ; SOARES, A. O. ; BAGGIO, A. J. . Potencial de aproveitamento medicinal de espécies do sub-bosque dos bracatingais da região de Curitiba, PR. Documentos, Colombo, p. 1-17, 2000.

NAPPO, M. E.; GRIFFITH, J. J.; MARTINS, S. V.; MARCO JUNIOR, P.; SOUZA, A. L.; OLIVEIRA FILHO, A. T. Dinâmica da estrutura fitossociológica da regeneração natural em sub-bosque de Mimosa scabrella Bentham em área minerada, em Poços de Caldas, MG. Revista Árvore, Viçosa, MG, v. 28, n. 6, p. 811-829, 2004.

NASCIMENTO, A. R. T.; LONGHI, S. J.; BRENA, D. A. Estrutura e padrões de distribuição espacial de espécies arbóreas em uma amostra de Floresta Ombrófila Mista em Nova Prata, RS. Ciência Florestal, Santa Maria, RS, v. 11, n. 1, p. 105-119, 2001.

NEGRELLE, R.A.B.; SILVA, F.C. da. Fitossociologia de um trecho de floresta com Araucaria angustifolia (Bert.) O. Ktze. no município de Caçador - SC. Boletim de Pesquisa Florestal, Colombo, n. 24/25, p. 37-54, 1992.

SEITZ, R. A.; JANKOVSKI, T. A regeneração natural de Pinus taeda. In: SIMPÓSIO FLORESTAL DO RIO GRANDE DO SUL, 5., 1998, Caxias do Sul. Anais do ... Caxias do Sul: Associação Gaúcha de Empresas Florestais (AGEFLOR), Sindicato das Indústrias da Madeira da Região Nordeste do Estado do Rio Grande do Sul (SINDIMADEIRA), Centro de pesquisas Florestais (CEPEF), Programa de PósGraduação em Engenharia Florestal da UFSM (PPGEF), 1998. p. 37-53.

STEIN, W.I. Developing an acceptable stoking survey. In: ANNUAL MEETING OF THE NORTHERN CALIFORNIA SECTION, SOCIETY OF AMERICAN FORESTERS, 1974, Oakland. Proceedings... Springfield: U.S. Department of Commerce, National Technical Information Service, 1974. p. 1-13.

WATZLAWICK, L.F.; SANQUETTA, C. R.; VALERIO, A.F.; SILVESTRE, R.; Caracterização da composição florística e estrutura de uma Floresta Ombrófila Mista, no Município de General Carneiro/PR. Ambiência; Guarapuava, v. 1, n. 2, p.229-238, 2005. 